\title{
Tests of achromatic phase shifters performed on the SYNAPSE test bench: a progress report
}

\author{
Pavel Gabor $^{a}$, Peter A. Schuller ${ }^{a}$, Bruno Chazelas ${ }^{b}$, Michel Decaudin ${ }^{a}$, Alain Labèque $^{a}$, \\ Philippe Duret ${ }^{a}$, Yves Rabbia ${ }^{c}$, Ralf Launhardt ${ }^{d}$, Jean Gay ${ }^{c}$, Zoran Sodnik ${ }^{e}$, Marc Barillot ${ }^{f}$, \\ Frank Brachet ${ }^{g}$, Thomas Laurent ${ }^{h}$, Sophie Jacquinod ${ }^{a}$, Denis Vandormael ${ }^{i}$, Jérôme Loicq ${ }^{i}$, \\ Dimitri Mawet ${ }^{j}$, Marc Ollivier $^{a}$, Alain Léger ${ }^{a}$ \\ ${ }^{a}$ Institut d'Astrophysique Spatiale, Univ. Paris-Sud XI, Orsay, France; \\ ${ }^{b}$ Observatoire de Genève, Genève, Switzerland; \\ ${ }^{c}$ Observatoire de la Côte d'Azur, Nice, France; \\ ${ }^{d}$ Max-Planck-Institut für Astronomie, Heidelberg, Germany; \\ ${ }^{e}$ European Space Agency, Nordwijk, Netherlands; \\ ${ }^{f}$ Thales Alenia Space, Cannes La Bocca, France; \\ ${ }^{g}$ Centre National d'Etudes Spatiales, Toulouse, France; \\ ${ }^{h}$ Univ. Liège, Liège, Belgium; \\ ${ }^{i}$ Centre Spatial de Liège, Liège, Belgium; \\ ${ }^{j}$ Univ. Liège, Institut d'Astrophysique et de Géophysique, Liège, Belgium
}

\begin{abstract}
The achromatic phase shifter (APS) is a component of the Bracewell nulling interferometer studied in preparation for future space missions (viz. Darwin/TPF-I) focusing on spectroscopic study of Earth-like exo-planets. Several possible designs of such an optical subsystem exist. Four approaches were selected for further study. Thales Alenia Space developed a dielectric prism APS. A focus crossing APS prototype was developed by the OCA, Nice, France. A field reversal APS prototype was prepared by the MPIA in Heidelberg, Germany. Centre Spatial de Liège develops a concept based on Fresnel's rhombs. This paper presents a progress report on the current work aiming at evaluating these prototypes on the SYNAPSE test bench at the Institut d'Astrophysique Spatiale in Orsay, France.
\end{abstract}

Keywords: Nulling interferometry, exoplanets, infrared spectroscopy, Darwin/TPF-I, achromatic phase shifters

\section{NULLING INTERFEROMETRY AND ACHROMATIC PHASE SHIFTERS}

Nulling interferometry, based on the concept suggested by Bracewell ${ }^{1}$ in 1978 , is one of the methods of future exoplanet research. It has been studied for the mission Darwin ${ }^{2}$ proposed to ESA as well as for the mission TPF-I (Terrestrial Planet Finder - Interferometry) ${ }^{3}$ proposed to NASA.

The basic performance parameter of a nulling interferometer is the "nulling ratio" (sometimes also referred to as "stellar leakage" although this term refers more properly to stray starlight due to the fact a star is not a point source) $n l(\lambda, t)=I_{\min } / I_{\max }$ where $I_{\min }$ and $I_{\max }$ stand for the intensity of the on-axis dark fringe and of the off-axis bright fringe, respectively.

The goal of Darwin/TPF-I is to perform infra-red $(6-18 \mu \mathrm{m})$ imagery of extrasolar planetary systems and spectroscopic observations of exoplanetary atmospheres in view of evaluating the presence of biomarkers. In order to reach this goal, the required nulling performance has been estimated ${ }^{4}$ as $\langle n l\rangle(\lambda)=1.010^{-5}(\lambda / 7 \mu \mathrm{m})^{3.37}$, with a level of long-term stability expressed as $\sigma_{\langle n l\rangle}(7 \mu \mathrm{m}, 10$ days $) \leq 310^{-9}$.

Corresponding author: p.gabor@jesuit.cz; Institut d'Astrophysique Spatiale, bât. 121, Campus Scientifique, 91405 Orsay, France; phone +33169858732. 
For a given geometric difference $\delta$ between the two optical paths, the corresponding phase difference $\Delta \varphi$ can be expressed as $\Delta \varphi=2 \pi \delta / \lambda$ where $\lambda$ is the wavelength. The phase shift therefore depends on the wavelength $\Delta \varphi=\Delta \varphi(\lambda)$. In order to obtain a wavelength-independent phase shift of $\pi$ at 0 OPD (optical path difference), an achromatic phase shifter (APS) has to be introduced into the setup. (An alternative approach, using an "adaptive nuller", has been studied at the JPL. ${ }^{5}$ )

Bracewell's nulling interferometer includes an APS as an optical means of eliminating the on-axis flux (stellar light), thus facilitating the detection of the off-axis flux (containing light from extrasolar planets). From the very inception, Darwin/TPF-I have opted for a Bracewell-based design, and therefore need an effective APS.

Many different concepts of achromatic phase shifters are available in the literature. ${ }^{6,7}$ Their merits need to be evaluated quantitatively. The Darwin collaboration studied ten APS concepts, ${ }^{8}$ promising $n l<10^{-6}$. The spectral range was that of $6-20 \mu \mathrm{m}$, with $6-18 \mu \mathrm{m}$ mandatory, the extension to $18-20 \mu \mathrm{m}$ priority number 2 , and that to $4-6 \mu \mathrm{m}$ priority number 3 . Four concepts were selected for further study:

1. Dispersive prisms (Fig. 1 a). The principle of this method consists in introducing dielectric plates into each arm of the interferometer. Their number, composition and thickness are optimised, together with the OPD, so that the chromaticity of the resulting phase shift in a given spectral band is below a specified level. As we shall see in the description of SYNAPSE (Sec. 2), a simple APS using two prisms of the same material in each arm of the interferometer is an integral part of the setup. A model with three prisms of three different materials per interferometer arm is under development by Thales Alenia Space.

2. Field reversal at reflection (Fig. 1 b). From two reflections, arranged in such a way that $s$ and $p$ polarisation components are successively permuted, the flip of field vectors provides an achromatic phase shift, as well as a pupil rotation of $\pi$. Suitably applied to a couple of interfering beams, a couple of periscope yields two lightwaves phase shifted by $\pi$ (opposed field vectors). Then a modified constructive interferometer provides two nulled outputs by suitably mixing the beams. A prototype APS was developed at Max-Planck-Institut für Astronomie in Heidelberg in collaboration with Kayser-Threde GmbH in Munich and the IOF Fraunhofer Institute for Applied Optics in Jena. ${ }^{9}$

3. Focus crossing (Fig. $1 \mathrm{c}$ ). The APS FC is based on the so-called focus-crossing (FC) effect where light undergoes a phase shift of $\pi$ when crossing a focus. The phenomenon is independent of wavelength. ${ }^{10,11}$ The principle of this approach is based on destructive interference. It is quite similar to the one of the Achromatic Interfero Coronagraph. ${ }^{12-15}$

APS FC comprises, one bearing two confocal half-parabolic off-axis mirrors, the other, two flat mirrors. The "confocal" module produces an achromatic $\pi$-phase shift, a pupil rotation by $\pi$ and an extra optical path (twice the focal length of a paraboloid), while the role of the "flat-flat" module is to balance this pathlength and to reproduce the beam geometry of the confocal module. At beam combination, the resulting amplitude is nulled for an on-axis point-like source, provided the wavefronts are perfectly cophased. If not, nulling is incomplete and a residual flux remains.

4. Fresnel's rhombs (Fig. $1 \mathrm{~d}$ ). are based on the phase shift at total internal reflection. It occurs when the incidence at material/air interface exceeds a limit angle $\vartheta_{\text {lim. }}$. A linear polarisation with azimuth $\pi / 4$ at entry, becomes a circular polarisation at exit (phase shift by $\pi / 2$ between $\mathrm{s}$ and p components). Two cascaded rhombs then yield a $\pi$ phase shift. A specific disposition of two coupled rhombs allows working with any azimuth of the polarisation at entry (dual polarisation is then allowed). Centre Spatial de Liège has been working on a concept combined with sub-lambda (subwavelength, i.e., zero order) gratings on the active surfaces of the rhombs. ${ }^{16,17}$ 


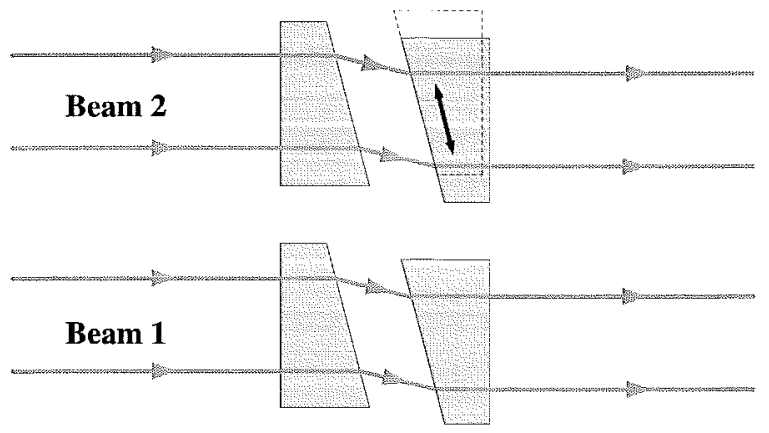

(a) Dielectric prisms



(c) Focus crossing

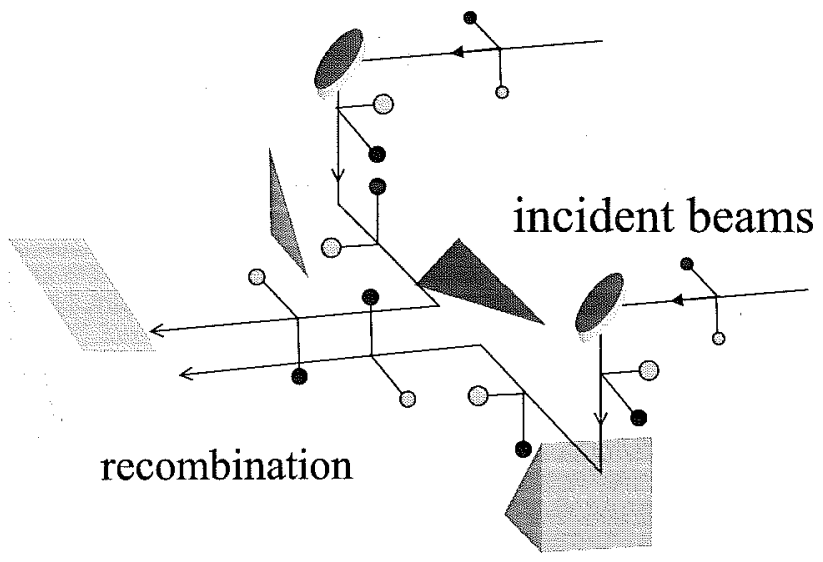

(b) Field reversal

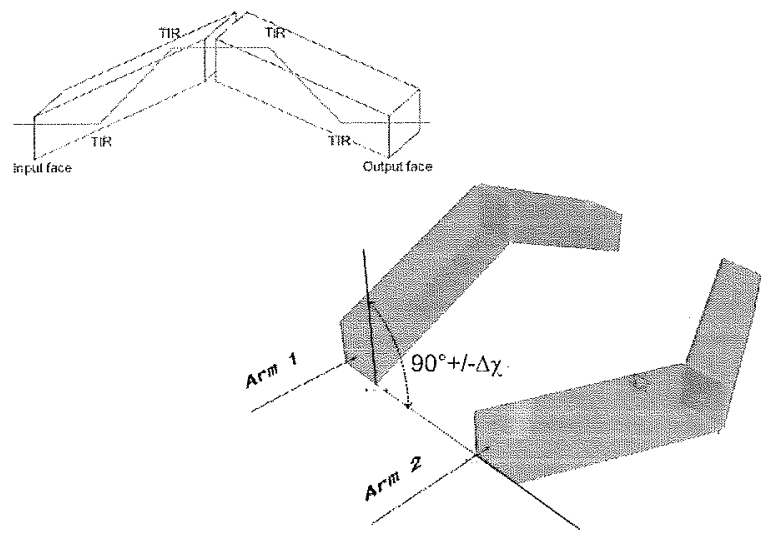

(d) Fresnel's rhombs

Figure 1. The four APS concepts under study for Darwin. 


\section{THE SYNAPSE TEST BENCH}

Since 1995 the Darwin team of the Institut d'astrophysique spatiale, Orsay, has been developping optical benches for high-contrast interferometry. The first test bench, operating at $10 \mu \mathrm{m}$, allowed to show that a monochromatic rejection factor of $10^{3}$ can be reached. ${ }^{18,19}$. Nonetheless, as we have already said, the real technical challenge is to maintain the stability of the nulling performance. Another test bench, SYmmetric Nuller for Achromatic Phase Shifters Evaluation (SYNAPSE), was designed for the research and development of this concept. ${ }^{20}$

\subsection{Sources}

We have been using three radiation sources:

$2000 \mathrm{~K}$ Black body source consisting of a LaCr hollow cylinder (20 mm diameter, $20 \mathrm{~cm}$ length) with a 5 $\mathrm{mm}$ hole on the side, conducting a current of $\approx 17 \mathrm{~A}$, emits visible and thermal radiation closely approximating a black body. The radiation is filtered using interference filters, focalised with an off-axis parabola, and injected into a single mode optical fiber (SMF; single-mode cutoff wavelength $\lambda_{c}=1.95 \mu \mathrm{m}$ or $2.85 \mu \mathrm{m}$ ) providing modal filtering. In order to avoid thermal and mechanical perturbations of the interferometer, the source subsystem, as well as the detector subsystem, are decoupled from the optical table.

HeNe laser at $3.39 \mu \mathrm{m}$ with the same filter, off-axis parabola and SMF as above. With a laser source, the role of the input SMF is effectively reduced to bringing the light from the source to the mechanically and thermically decoupled interferometer.

"White laser" or "supercontinuum" source based on a non-linear, photonic crystal optical fiber, and producing up to $3 \mathrm{~mW} / \mathrm{nm}$ in the range $400-2500 \mathrm{~nm}$. After filtering, the source output is coupled with the same SMF as above, using a spherical mirror.

\subsection{Interferometer}

The interferometer is set up on an optical table with passive vibration protection, and protected from acoustic and thermal perturbations by a plexiglass box. The optical bench itself comprises two modified Mach-Zehnder interferometers serving as beam-splitting and beam-recombination devices ${ }^{21}$ (Fig. 2), creating the two identical beams, delay lines, and a simple APS with two $\mathrm{CaF}_{2}$ prisms in each interferometer arm.

Both delay lines, based on rooftop mirrors, are motorised: one with a stepper motor running $25 \mathrm{~mm}$ (i.e., optical path of $50 \mathrm{~mm}$ ) at $0.1 \mu \mathrm{m}$ reproducibility, and the other with a piezoelecric actuator running $15 \mu \mathrm{m}$ at a reproducibility better than $0.5 \mathrm{~nm}$.

The beam-splitter and beam-combiner systems use parallel $\mathrm{CaF}_{2}$ plates. Their thickness is not guaranteed to be identical to the required accuracy, and therefore a compensator needs to be included in the design. The bench's intrinsic APS, comprising two $\mathrm{CaF}_{2}$ prisms in each interferometer arm, fulfills this role. As an APS, it can introduce any given phase shift, and reduces the chromaticity in the $\mathrm{K}$ or $\mathrm{L}$ band so that the resulting theoretical nulling performance is better than $\approx 10^{-5}$.

Since the bench (if well aligned) is symmetric by design, the flux of the two arms is balanced using simply injection into the exit SMF, tweaking the orientation of the folding mirror illuminating the off-axis parabola. The better the superposition of the two beams, the easier the balancing.

For the tests of APS prototypes, the two identical beams pass through the tested modules before entering the beam-combiner subsystem. In the current configuration, we use a translation stage with folding mirrors as an optical switch to insert the hosted APS FC prototype into the optical path. 


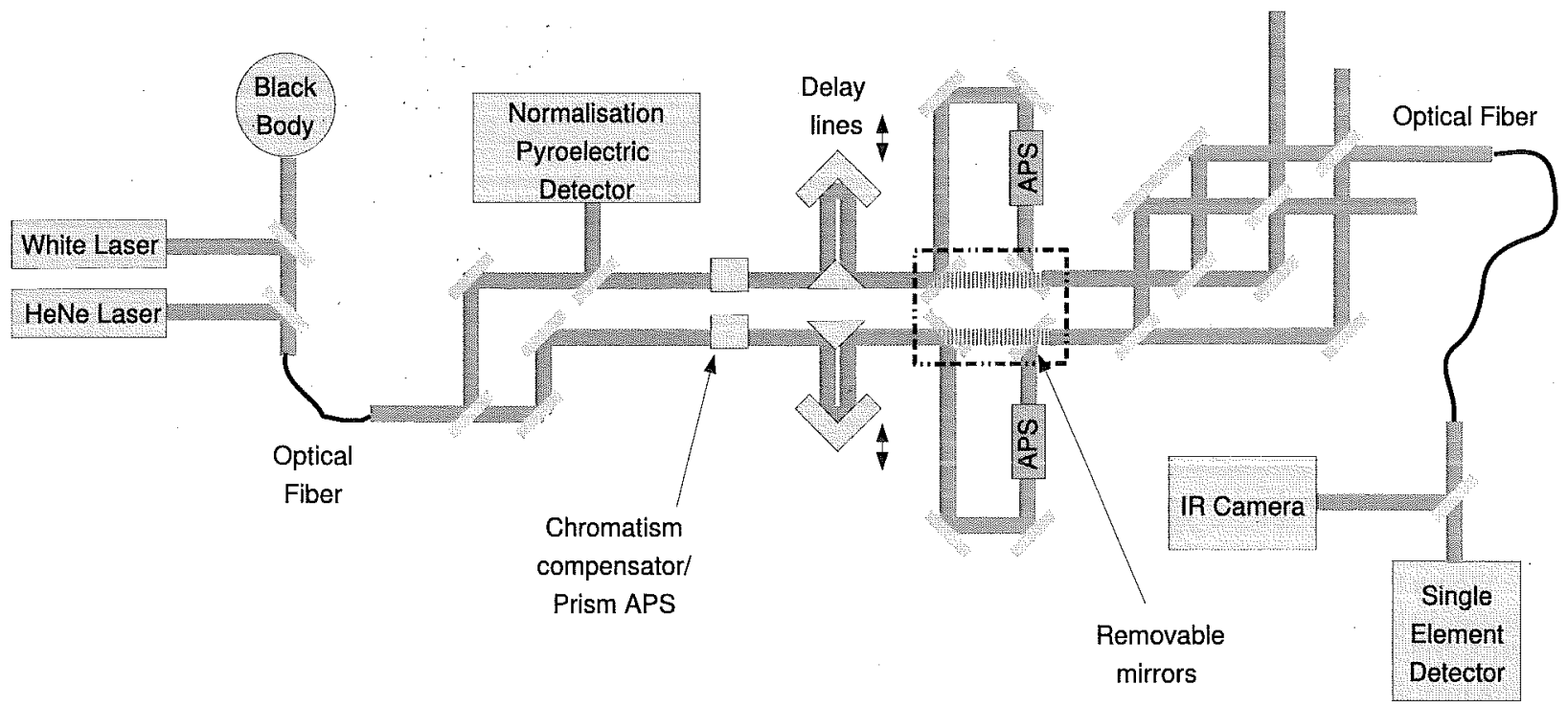

Figure 2. SYNAPSE (from left to right): Sources (three options), SMF, beam-splitter subsystem, $\mathrm{CaF}_{2}$ prisms (chromatism compensator and/or APS), optical switch, APS prototype modules, beam-combiner subsystem, SMF, detectors (two options).

\subsection{Detectors}

In order to normalise the flux as measured at the output of the bench (useful also for fine-tuning of beam injection into the SMF), we have installed a simple pyroelectric detector at one of the redundant outputs of the beam-splitter subsystem.

A SMF, placed at the exit of the interferometer, provides modal filtering. Two types of detectors are used:

Single element detector is a commercial, liquid nitrogen cooled, InSb detector. Its signal passes through a lock-in amplifier before being digitised.

Array detector is an astronomical camera system previously used on the CFHT. A $128 \times 128$ pixel InSb array is mounted in a solid nitrogen cooled cryostat.

\section{TESTS AND PRELIMINARY RESULTS}

\subsection{Evolution}

The work of Frank Brachet ${ }^{20}$ has documented two features of the SYNAPSE nulling performance in the $\mathrm{K}$ band $(2-2.5 \mu \mathrm{m})$. The first being the best value of a nulling ratio of $n l=1.510^{-4}$. The second parameter observed was the degree of nulling performance stability. Let us reproduce the results of two typical acquisitions. The first (Fig. 3, left) shows an acquisition of about $200 \mathrm{~s}$ with a mean nulling ratio $\langle n l\rangle=2.710^{-4}$ with a considerable standard deviation $\sigma_{\langle n l\rangle}=610^{-5}$. The second (Fig. 3, right) is a slightly longer recording, of about 10 minutes 

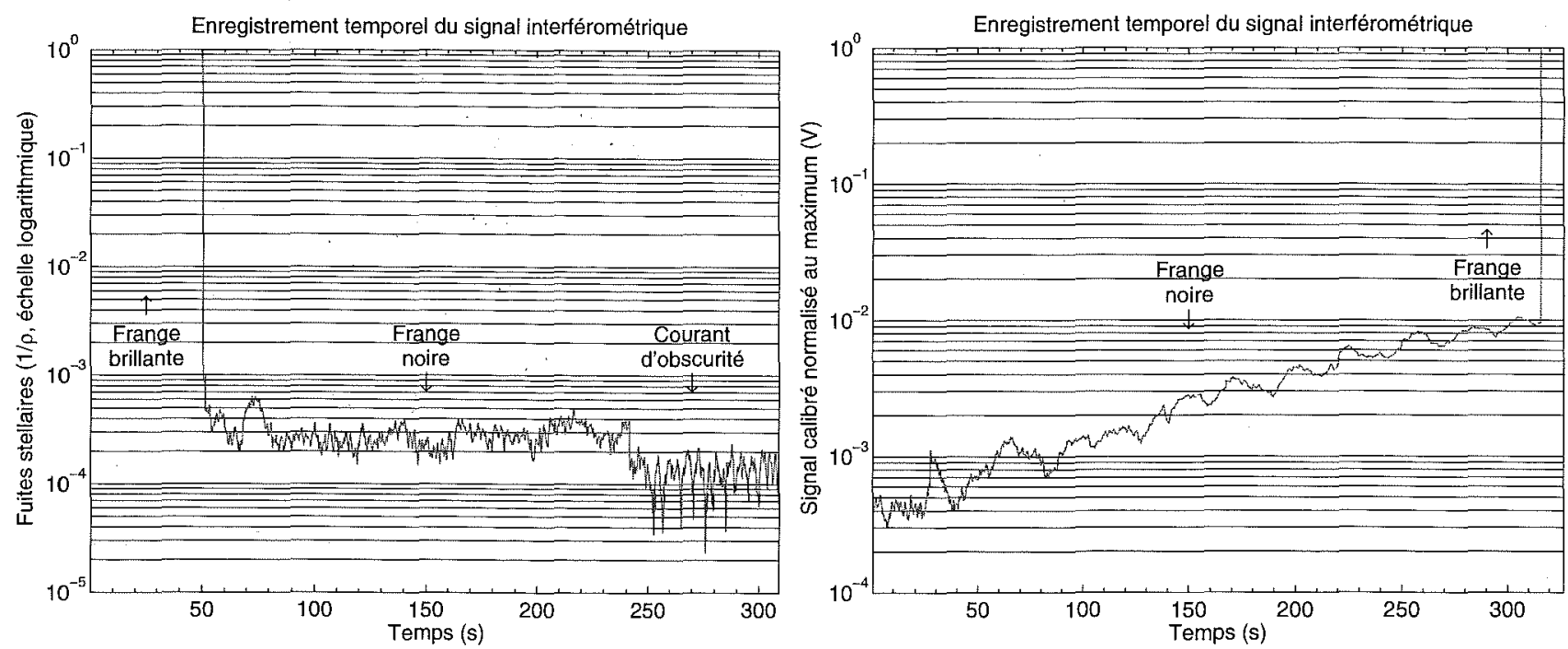

Figure 3. SYNAPSE measurements in the K spectral band. Left: The transition between the bright fringe (the first 50 seconds) and the dark fringe (between $50 \mathrm{~s}$ and $240 \mathrm{~s}$ ) was performed manually by modifying the length of the delay line. The last part of the curve shows the dark current (the detector being covered). The mean rejection factor is about 2.4 times greater than the dark current of the detector. Right: Same measurement with a significant drift.

with a nulling ratio $\langle n l\rangle=2.510^{-4}$ during the first 100 seconds (with $\sigma_{\langle n l\rangle}=610^{-5}$ ), showing the effects of significant drift, probably due to OPD instability.

In order to stabilise the bench, we have introduced a control system for OPD, ${ }^{22}$ implenting dithering (periodic changes) of the OPD close to the optimal value. A classical form of this technique has been investigated, ${ }^{18}$ although this first experiment was inconclusive. More recently, Schmidtlin et al. ${ }^{23}$ have demonstrated the potential of dithering in a sequential way, obtaining good levels of stabilisation with nulling ratio around $810^{-7}$ with a laser diode at $638 \mathrm{~nm}$. It was this work that provided our team with decisive inspiration.

Implementing this approach, we have succeeded not only in stabilising the nulling performance, but also gained a powerful instrument for bench optimisation (cf. Sec. 3.2).

In the $\mathrm{K}$ band, we have obtained $\langle n l\rangle=310^{-4}$ and $\sigma_{\langle n l\rangle}(3 \mathrm{~s})=210^{-4}$ at integration times of $3 \mathrm{~s}$, which improves to $\sigma_{\langle n l\rangle}(2 \mathrm{hrs})=810^{-6}$ after 2 hours of integration. Since $\sigma$ in Fig. 4 is consistent with $\tau^{-1 / 2}$ behaviour up to $\tau \approx 500 \mathrm{~s}$, with $\sigma_{\langle n l\rangle}(500 \mathrm{~s}) \approx 1.510^{-5}$, we extrapolate that if drifts were kept at bay we would obtain $\sigma_{\langle n l\rangle}(10$ days $)=310^{-7}$ after an integration of 10 days, still two orders of magnitude short of the goal.

In order to find ways of improving the nulling performance of the bench, we started working with monochromatic light, at $3.39 \mu \mathrm{m}$, produced by a HeNe laser. The first results were limited to $\langle n l\rangle=610^{-5}$. Introducing polarisers has led to $\langle n l\rangle=110^{-5}$ (an OPD scan is shown in Fig. 5). This result is coherent with our theoretical studies ${ }^{24}$ of the effects of polarisation mismatch (primarily due to residual alignment errors) on the nulling performance.

OPD-stabilised long-duration measurements (the OPD dithering was able to follow the dark fringe over a period $>11 \mathrm{hrs}$ ) have shown, at this level of nulling, instabilities that cannot be fully controlled with OPD (Fig. 5, right). Note that that the system remains at the "bottom" of the dark fringe (best nulling-ratio values) for typically $10 \mathrm{~min}$ although an analysis of the noise of the acquired data (plotting standard deviations of the running average of the nulling ratio against the width of the averaging window) exposes deviations from the $\tau^{-1 / 2}$, white-noise behaviour.

The next step towards full stabilisation of the bench would have to involve control of flux mismatch between the interferometer arms. In order to perform tests of APS prototypes at $n l=10^{-5}$, however, OPD stabilisation suffices in providing reliable and reproducible results. 

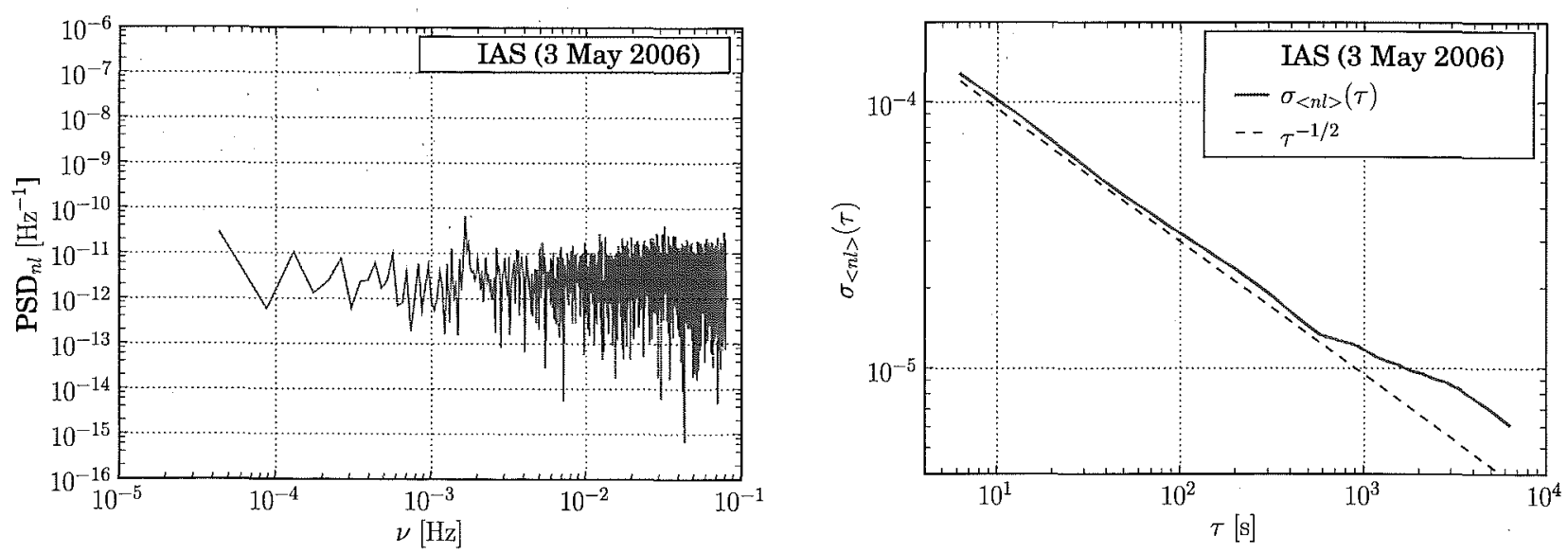

Figure 4. SYNAPSE measurements in the K spectral band. Left: Power spectral density of the nulling function, $\operatorname{PSD}(n l)$. Note that $1 / f$ component is negligible. Right: Standard deviations of the running average of the nulling function over the time interval $\tau, \sigma_{\langle n l\rangle}(\tau)$ (curve above). A (displaced) $\tau^{-1 / 2}$ function is shown for comparison (line below). Note that up to $\tau \approx 500 \mathrm{~s}$, the experimental curve is consistent with the $\tau^{-1 / 2}$ behaviour, with $\sigma_{\langle n l\rangle}(500 \mathrm{~s}) \approx 1.510^{-5}$.
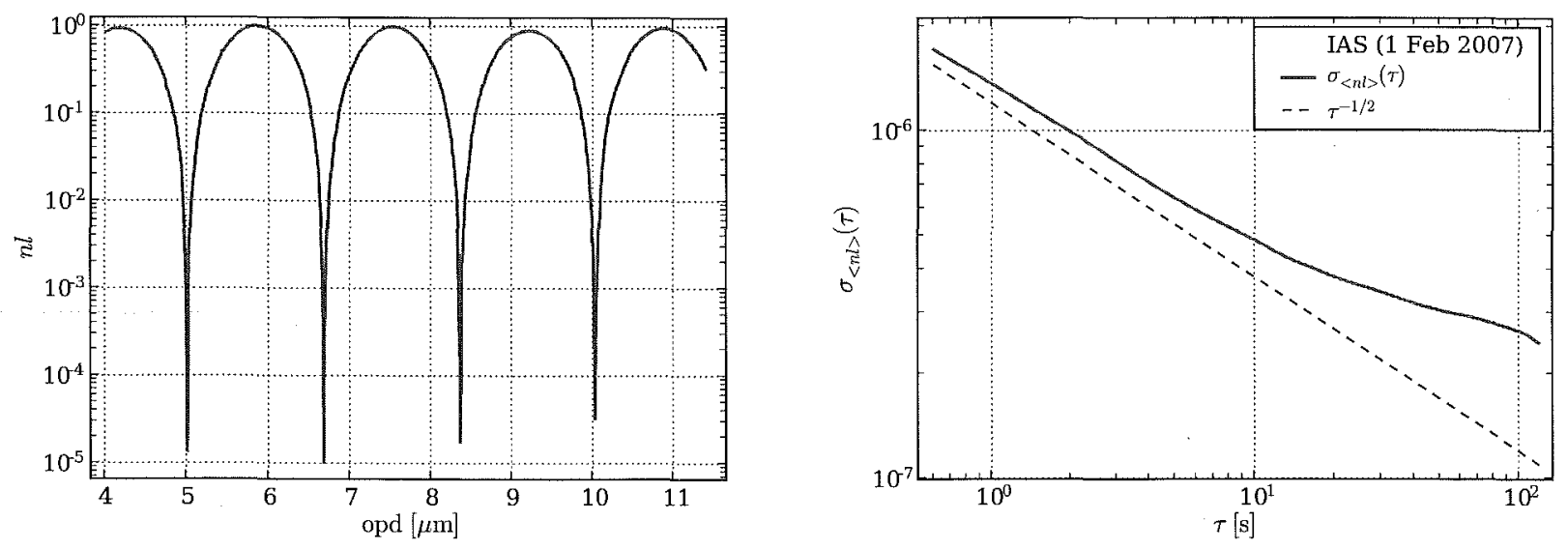

Figure 5. Measurements with a HeNe laser at $3.39 \mu \mathrm{m}$. Left: Results of an OPD scan in terms of the nulling ratio $n l$. Right: Results of a 10-minute OPD-stabilised acquisition of the signal at the "bottom" of the dark fringe presented in terms of standard deviations of the running average of the nulling function over the time interval $\tau, \sigma_{\langle n l\rangle}(\tau)$. A (displaced) $\tau^{-1 / 2}$ function is shown for comparison (line below). 


\begin{tabular}{|l|l|c|c|c|l|}
\hline & $\lambda$ & polarisers & APS FC & $n l$ & stability \\
\hline \hline Nor '05 & $\mathrm{K}$ band & - & - & $1.510^{-4}$ & drifts \\
\hline May '06 & $\mathrm{K}$ band & - & - & $510^{-4}$ & stable (3 hrs) \\
\hline Jan '07 & $3.39 \mu \mathrm{m}$ & - & - & $10^{-4}$ & \\
\hline Jan '07 & $3.39 \mu \mathrm{m}$ & + & - & $10^{-5}$ & stable (10 min) \\
\hline Feb '08 & $3.39 \mu \mathrm{m}$ & + & - & $10^{-5}$ & \\
\hline Feb '08 & $3.39 \mu \mathrm{m}$ & + & + & $10^{-5}$ & \\
\hline Feb '08 & K band & - & + & $410^{-4}$ & \\
\hline Feb '08 & K band & + & + & $410^{-4}$ & \\
\hline
\end{tabular}

Table 1. An overview of progress on SYNAPSE in terms of $n l$ and stability (when available).

After these encouraging results with monochromatic light we concentrated our efforts on improving the level of null in broad band. From the results of monochromatic measurements we deduced that the factor limiting nulling levels of the test bench could not be thermal and mechanical (vibrations) stability. We have also seen that improving bench alignement (to within $<10$ arcsec as opposed to the previous $<3$ arcmin) may help reduce polarisation mismatch, greatly facilitating flux balancing of the two interferometer arms. An overview of our progress in terms of $n l$ and stability (when available) is given in Tab. 1. Re-aligning the bench and accompanying measurements performed also enabled us to eliminate other hypotheses, namely,

- vignetting, mismatched and/or non-gaussian beam profiles: checked in the visible with cameras, and in the IR with pinhole scans;

- lateral shift and/or angular misalignment of beams injected into the exit fiber, relative to each other and/or to the fiber's eigen mode: checked in the visible to $<0.1 \mathrm{~mm}$ and $<10 \operatorname{arcsec}$, while calculations tell us that we should be at $n l \approx 1^{-6}$ (considering lateral and radial beam chromaticity) while the influence of the surface quality of the off-axis parabola is negligible when the beams are superimposed as specified (otherwise a flux mismatch of $>30 \%$ between the two beams would be observed);

- incorrect working point idendification of the bench's intrinsic APS (dielectric prisms): different working points, corresponding to a combination of OPD and of differential thickness of the dielectric in the arms of the interferometer, may give similar fringe patterns (because they correspond to phase shifts of $(2 n-1) \pi$ ) but the optimal (in terms of providing the best nulling performance) working position is unique; with a degraded null it is possible to mistake $a$ working point for the working point; we checked this, measuring $n l$ at several such working points, ranging from $-11 \pi$ to $+11 \pi$;

- spectral mismatch of the two interfering beams: measuring the each beam with a spectrometer yielded a mismatch estimate that would lead to $n l<3.810^{-5}$;

- asymetry in beam-splitter coatings: assuming differerences in thickness of $10 \%$ we calculated $n l<10^{-6}$;

- inhomogeneities in the material of the bench's intrinsic APS: checked interferometrically before APS production; we calculated that such inhomogeneities would lead to $n l<10^{-6}$.

\subsection{Techniques}

Several measurement techniques have been studied for SYNAPSE:

- Placing an array detector with a dispersive prism at the exit of the bench while scanning OPD, provides a series of low-resolution spectra. In an ideal case the resulting pattern would contain a straight dark fringe. Its deviation from the ideal form yields a measure of phase-shift chromaticity. ${ }^{24}$ The chief advantages of this approach is that it is inherently independent of flux mismatch, and that it works even when the 
signal-to-noise ratio does not allow for a direct measurement of $n l$. Nonetheless, we have not implemented this approach very widely. One difficulty has to do with OPD drifts, and the other is practical: our camera is much more difficult to use than the single element detector.

- Another technique which is under study is Fourier transform of OPD scans with the coarse long line. ${ }^{24}$ The beams are recombined in a SMF which also provides modal filtering, and the flux is measured using a single element detector. The Fourier transform method is robust vis-à-vis chromatic flux mismatch. In SYNAPSE, an improved delay-line actuator would greatly facilitate the application of this technique.

- The measurements presented in this paper were obtained following an experimental protocol which we have been developping for SYNAPSE with the single mode detector (as described in the previous point). Note that OPD stabilisation is used not only to reach and maintain the optimal OPD (and therefore enabling reproducible measurements of $n l$ and $\sigma_{n l}$ ) but also tweaking experiments where the impact of small changes in flux balance, $\mathrm{CaF}_{2}$ prism position, monochromator prism orientation, etc. on the $n l$ and OPD can be monitored.

- Check position of optical switch: experiment with or without FC APS.

- Check $\mathrm{CaF}_{2}$ prism and long delay-line position.

- Start optimising injection into the exit fiber by maximising Beam 1 flux with motorised folding mirrors.

- Switch to Beam 2. Remove residual flux mismatch by tweaking folding mirror orientation (aiming for $\Delta I / I \approx 0.5 \%)$.

- Perform OPD scan with the fine delay line. Find current dark fringe position and the first $n l$ value.

- Repeat OPD scan, focusing on the dark fringe. Obtain a measure of $n l$.

- Start OPD-stabilised DAQ, monitoring the flux and OPD position.

- Perform tweaking experiments:

* tweak folding-mirror position in order to monitor/minimise the impact of flux mismatch on $n l$;

* while monitoring its impact on $n l$, tweak $\mathrm{CaF}_{2}$ prism position in order to fine-tune the prism APS;

* shift the working wavelength by rotating the monochromator prism, while OPD-stabilisation follows the dark fringe in order to measure corresponding OPD shift.*

\subsection{Prospective}

Pertinent measurements of APS performance on the SYNAPSE test bench will be possible once improved nulling levels are reached. Identifying the limiting factors is therefore our immediate goal. The tests in progress concentrate on the single-mode filtering performance of the optical fibers we are using. In a setup like SYNAPSE, SMF's are crucial in bridging over the problem of wavefront bumpiness induced by errors of optical surfaces. ${ }^{25}$ However, the quality of modal filtering provided by a given SMF is hard to assess. ${ }^{26}$ The work in progress includes

- measurements in a narrow spectral band (16 nm) around $2.3 \mu \mathrm{m}$,

- study of secondary-mode rejection by modifying fiber curvature,

- measurements in the $\mathrm{L}$ band $3-4 \mu \mathrm{m}$, filtering with a single-mode fiber with $\lambda_{c}=1.95 \mu \mathrm{m}$,

- measurements using a prism-based monochromator.

\footnotetext{
${ }^{*}$ This paper does not contain results obtained using this technique.
} 


\section{DISCUSSION AND CONCLUSION}

The primary objective of this paper was to present an update on our progress with the SYNAPSE test bench. Our work has so far demonstrated that the setup reaches a well reproducible monochromatic nulling level $n l=10^{-5}$ at $3.39 \mu \mathrm{m}$ which can be maintained for 10 minutes, thus reaching stability of $\sigma_{\langle n l\rangle}(3.39 \mu \mathrm{m}, 100 \mathrm{~s}) \leq 410^{-7}$. The reproducible nulling level in the $\mathrm{K}$ band is currently of $n l=310^{-4}$. We have verified that it can be maintained for at least several hours, reaching stability levels of $\sigma_{\langle n l\rangle}(2.2 \mu \mathrm{m}, 2 \mathrm{hrs})=810^{-6}$.

Regarding evaluation of APS prototypes, at the moment, the bench allows for work with its "intrinsic" APS (a pair of dielectric prisms per branch) and with the APS FC. The current primary objective of experiments with the APS FC is to provide us with more options for our diagnostic tests. The two APS concepts are in fact very different (one flips the field of view, while the other does not, one works only in a set spectral band outside of which nulling levels deteriorate fast, the other based on a physical principle which is independent of wavelength), and therefore using both gives us more information about the bench itself.

The APS FC was initially built to work in the spectral range of $6-18 \mu \mathrm{m}$ (proposed range for Darwin/TPFI), and its expected nulling performance is $n l \leq 10^{-6}$, depending on the bench configuration. In the working conditions of SYNAPSE the expected value is $10^{-5}$. Up to now this performance has been demonstrated with laser light at $3.39 \mu \mathrm{m}$. Rejection performance of the APS FC in the $\mathrm{K}$ band $(2-2.5 \mu \mathrm{m})$ is to date limited by the performance of the SYNAPSE bench itself.

Although evaluation of the performance of different APS's may be done using fringe dispersion or a monochromator (phase-shift measurement as a function of wavelength), clearly the most representative type of APS estimate calls for measurements with broad-band polychromatic light. We are making progress towards eliminating all possible limiting factors to SYNAPSE broad-band performance. The work is ongoing and we shall present an update at the SPIE Congress.

\section{ACKNOWLEDGMENTS}

This work was supported by Centre National d'Etudes Spatiales (action RET, n. R-506/SU-002-022) and Agence Nationale de la Recherche (thème blanc, n. 5A 0617).

\section{REFERENCES}

[1] Bracewell, R. N., "Detecting nonsolar planets by spinning infrared interferometer," Nature 274, 780-(Aug. 1978).

[2] Karlsson, A. and Kaltenegger, L., "The technology of DARWIN," in [ESA SP-539: Earths: DARWIN/TPF and the Search for Extrasolar Terrestrial Planets], Fridlund, M., Henning, T., and Lacoste, H., eds., 41-46 (Oct. 2003).

[3] Coulter, D. R., "NASA's Terrestrial Planet Finder mission: the search for habitable planets," in [ESA SP539: Earths: DARWIN/TPF and the Search for Extrasolar Terrestrial Planets], Fridlund, M., Henning, T., and Lacoste, H., eds., 47-54 (Oct. 2003).

[4] Chazelas, B., Brachet, F., Bordé, P., Mennesson, B., Ollivier, M., Absil, O., Labèque, A., Valette, C., and Léger, A., "Instrumental stability requirements for exoplanet detection with a nulling interferometer : variability noise is a central issue," Applied Optics 45(5), 984-992 (2006).

[5] Peters, R. D., Lay, O. P., Hirai, A., and Jeganathan, M., "Adaptive nulling for the Terrestrial Planet Finder Interferometer," in [Advances in Stellar Interferometry, Proceedings of SPIE Volume 6268.], Monnier, J. D., Schöller, M., and Danchi, W. C., eds., 62681C-1-7 (2006).

[6] Rabbia, Y., Gay, Y., Bascou, E., and Schneider, J. L., "Summary assessment report on achromatic phase shifters for space interferometry," tech. rep., ESA ESTEC, Contract 14 398/00/NL/MV (2001).

[7] Rabbia, Y., Gay, Y., and Bascou, E., "Achromatic phase shifters for nulling interferometry," in [ICSO'2000 - Conférence Internationale d'Optique Spatiale, Toulouse, France, Dec. 5-7, 2000) CNES Eds.], 389-400 (2000).

[8] "Review and discussion of APS requirements, ESA AO/1-3946/02/NL/JA," tech. rep. (2002). 
[9] Launhardt, R., "Breadboarding an achromatic phase shifter for midinfrared nulling interferometry," To be presented at the Society of Photo-Optical Instrumentation Engineers (SPIE) Conference 7013, paper 7013-175 (June 2008).

[10] Gouy, G. C.R. Acad.Sci. Paris 110, 1251 (1890).

[11] Boyd, R. W. JOSA 70, 877 (1980).

[12] Gay, J. and Rabbia, Y. C.R. Acad.Sci. Paris IIb, 322, 265 (1996).

[13] Baudoz, P., Rabbia, Y., and Gay, J., "Achromatic interfero coronagraphy I. Theoretical capabilities for ground-based observations," Astronomy and Astrophysics 141, 319-329 (Jan. 2000).

[14] Baudoz, P., Rabbia, Y., Gay, J., Burg, R., Petro, L., Bely, P., Fleury, B., Madec, P.-Y., and Charbonnier, F., "Achromatic interfero coronagraphy. II. Effective performance on the sky," Astronomy and Astrophysics 145, 341-350 (Aug. 2000).

[15] Rabbia, Y., Gay, J., Chazelas, B., Labèque, A., and Rivet, J.-P., "Nulling interferometry : Lommel's integrals applied to a Fresnel's diffraction effect," in [IAU Colloq. 200: Direct Imaging of Exoplanets: Science 63 Techniques], Aime, C. and Vakili, F., eds., 265-270 (2006).

[16] Mawet, D., Lenaerts, C., Riaud, P., Vandormael, D., Loicq, J., Verstraeten, D., Fleury, K., Habraken, S., and Surdej, J., "Infrared achromatic phase shifters using modulated total internal reflection," in [Advances in Stellar Interferometry. Edited by Monnier, John D.; Schöller, Markus; Danchi, William C.. Proceedings of the SPIE, Volume 6268, pp.62682J (2006).1, Presented at the Society of Photo-Optical Instrumentation Engineers (SPIE) Conference 6268 (July 2006).

[17] Mawet, D., Hanot, C., Lenaers, C., Riaud, P., Defrère, D., Vandormael, D., Loicq, J., Fleury, K., Plesseria, J.-Y., Surdej, J., and Habraken, S., "Fresnel rhombs as achromatic phase shifters for infrared nulling interferometry," Opt. Express 15(20), 12850-12865 (2007).

[18] Ollivier, M., Mariotti, J.-M., Léger, A., Sékulic, P., Brunaud, J., and Michel, G., "Interferometric coronography for the DARWIN space mission - Laboratory demonstration experiment," Astronomy and Astrophysics 370, 1128-1136 (May 2001).

[19] Ollivier, M., Coronographie interférentielle pour la mission Darwin, PhD thesis, Université Paris XI (Dec. 1999).

[20] Brachet, F., Etude et développement d'un déphaseur achromatique pour l'interféromtrie en frange noire, PhD thesis, Paris XI (2005).

[21] Serabyn, E. and Colavita, M. M., "Fully Symmetric Nulling Beam Combiners," Applied Optics 40, 16681671 (Apr. 2001).

[22] Gabor, P., Chazelas, B., Brachet, F., Ollivier, M., Decaudin, M., Jacquinod, S., Labèque, A., and Léger, A., "Stabilising a nulling interferometer using optical path difference dithering," Astronomy \& Astrophysics 483, 365-369 (May 2008).

[23] Schmidtlin, E., Wallace, J. K., Samuele, R., Levine, B. M., and Shao, M., "Recent Progress of Visible Light Nulling Interferometry and First 1 Million Null Result," in [Direct Imaging of Exoplanets: Science and Techniques], Aime, C. and Vakili, F., eds., 353-360, IAU Colloquium No. 200 (2005).

[24] Chazelas, B., Contribution à l'étude de l'interférométrie annulante pour la détection d'exo-planètes, $\mathrm{PhD}$ thesis, Paris XI (2007).

[25] Mennesson, B., Ollivier, M., and Ruilier, C., "Use of single-mode waveguides to correct the optical defects of a nulling interferometer," Optical Society of America Journal 19, 596-602 (Mar. 2002).

[26] Ksendzov, A., Lay, O., Martin, S., Sanghera, J. S., Busse, L. E., Kim, W. H., Pureza, P. C., Nguyen, V. Q., and Aggarwal, I. D., "Characterization of mid-infrared single mode fibers as modal filters," Applied Optics 46, 7957-7962 (Nov. 2007). 